\title{
Minkowskian Dynamics of a Polyakov Loop Model under a Heating Quench
}

\section{Bernd A. Berg*}

Department of Physics, Florida State University, Tallahassee, FL 32306, USA

\section{Alexei Bazavov ${ }^{\dagger}$}

Department of Physics, University of Arizona, Tucson, AZ 85721, USA

\section{Adrian Dumitru}

Department of Natural Sciences, Baruch College, CUNY, 17 Lexington Av, New York, NY 10010; RIKEN BNL Research Center, Brookhaven National Lab, Upton, NY 11973

\begin{abstract}
We report on a recent calculation of the time evolution of Polyakov loop fluctuations from a simple classical relativistic Lagrangian after a quench into the deconfined phase of SU(3) gauge theory. The structure factors indicate spinodal phase conversion followed by relaxation, similarly to results obtained via Markov Chain Monte Carlo techniques in SU(3) lattice gauge theory. The time for which the structure factor reaches its maximum diverges in the long-wavelength limit due to formation of non-perturbatively large competing Z(3) Polyakov loop domains, which delay thermalization of long wavelength modes. For realistic temperatures, and away from the extreme weak-coupling limit, also modes with $k$ on the order of $T$ experience delayed thermalization. As relaxation times of very long wavelength modes are found to be on the order of the size of the system, the dynamics of competing domains should accompany the hydrodynamic description of the deconfined vacuum.
\end{abstract}

The XXVI International Symposium on Lattice Field Theory

July 14 - 19, 2008

Williamsburg, Virginia, USA

\footnotetext{
*Speaker.

${ }^{\dagger} \mathrm{AB}$ replaced $\mathrm{BB}$ to deliver the talk.
} 


\section{Introduction}

Relativistic Heavy-Ion Collision experiments carried out at Brookhaven National Laboratory provide support for the existence of a quark-gluon plasma phase of QCD [1]. A collision of two heavy nuclei at high energy releases a large number of gluons from the wave functions of the colliding nuclei [2]. Those gluons interact and eventually form a thermalized QCD plasma with a temperature in excess of the critical temperature for deconfinement. Complete (and consistent) theoretical understanding of the thermalization process is presently lacking. Baier, Mueller, Schiff and Son developed the so-called "bottom-up" approach [䚮, which is a framework for understanding the processes leading to thermalization and for calculating the thermalization time of the QCD medium as well as its initial temperature (see, however, the critique in Ref. [4]).

The "bottom-up" approach is based on solving the Boltzmann equation for quasi-particles in a trivial vacuum and neglects the structure of the deconfined phase of the non-Abelian gauge theory arising from the Z(3) center symmetry discovered by t' Hooft and Polyakov [5] and further studied in [6]. Below we report results of Ref. [7], which show that a relativistic model allows for non-perturbatively large variations of Polyakov loops in space, and domain walls form separating regions of different $\mathrm{Z}(3)$ orientation. The competition between such domains affects the thermalization of long-wavelength modes of the Polyakov loop and some model estimates for the relevant wave lengths and time scales are provided.

We adopt a simplified picture where a relativistic heavy-ion collision is viewed as a quench that instantly heats the system to a temperature above the deconfining temperature. The response of Polyakov loop Structure Factors (SFs) to such a heating quench has been studied in SU(3) LGT by Markov Chain Monte Carlo (MCMC) simulations for Glauber (dissipative) dynamics [8]. As unambiguous signals for the transition one finds a dynamical growth of the SF, reaching maxima which scale approximately with the volume, a behavior characterizing spinodal phase conversion [9]. Relaxation to the vacuum ensemble at high temperature becomes feasible only after each SF has overcome its maximum value. For SU(3) gauge theory this relaxation time diverges with increasing system size due to competing order-order domains with different $Z$ (3) center group triality. Hence, one should not a-priori exclude the possibility that, under heating, the long wavelength modes in the system do not equilibrate but instead get stuck in the neighborhood of the SF maxima.

Glauber (model A) dynamics is expected to describe the dissipative features of the transition from one equilibrium ensemble to another well. Such SU(3) MCMC simulations converge to 3D equilibrium ensembles, which are the same as in Minkowski space, because the fourth extension of the Euclidean lattice serves only to define the temperature. The major drawback of Glauber dynamics is not the 4D Euclidean formulation but that it is non-relativistic and, more importantly, that one does not know how to connect the MCMC updating step to a physical time scale. In this talk we report results [7] from heating quenches into the deconfined phase within Pisarski's [10] relativistic effective model of Polyakov loops (compare also models of Ref. [11]).

\section{Effective Model of Polyakov Loops}

In Pisarski's model [10] the deconfined phase of a pure gauge theory is described as a condensate of Polyakov loops. The Z(3)-symmetric effective potential for Polyakov loops $\ell$ (complex for 
$\mathrm{SU}(3))$ with cubic and quartic interactions takes the form

$$
\mathscr{V}(\ell)=\left(-\frac{b_{2}}{2}|\ell|^{2}-\frac{b_{3}}{6}\left(\ell^{3}+\left(\ell^{*}\right)^{3}\right)+\frac{1}{4}\left(|\ell|^{2}\right)^{2}\right) b_{4} T^{4} .
$$

The energy scale is set by $T^{4}$. The mass coefficient $b_{2}=b_{2}(T)$ is temperature dependent, while $b_{3}$ and $b_{4}$ are constants. These couplings are chosen to reproduce lattice data for the $\mathrm{SU}(3)$ pressure and energy density above $T_{c}$. At temperatures $T_{f}>T_{c}$ the effective potential takes the shape shown in Fig. 1. We show equal height contours in the $\left(\ell_{r}, \ell_{i}\right)$ plane of this figure.

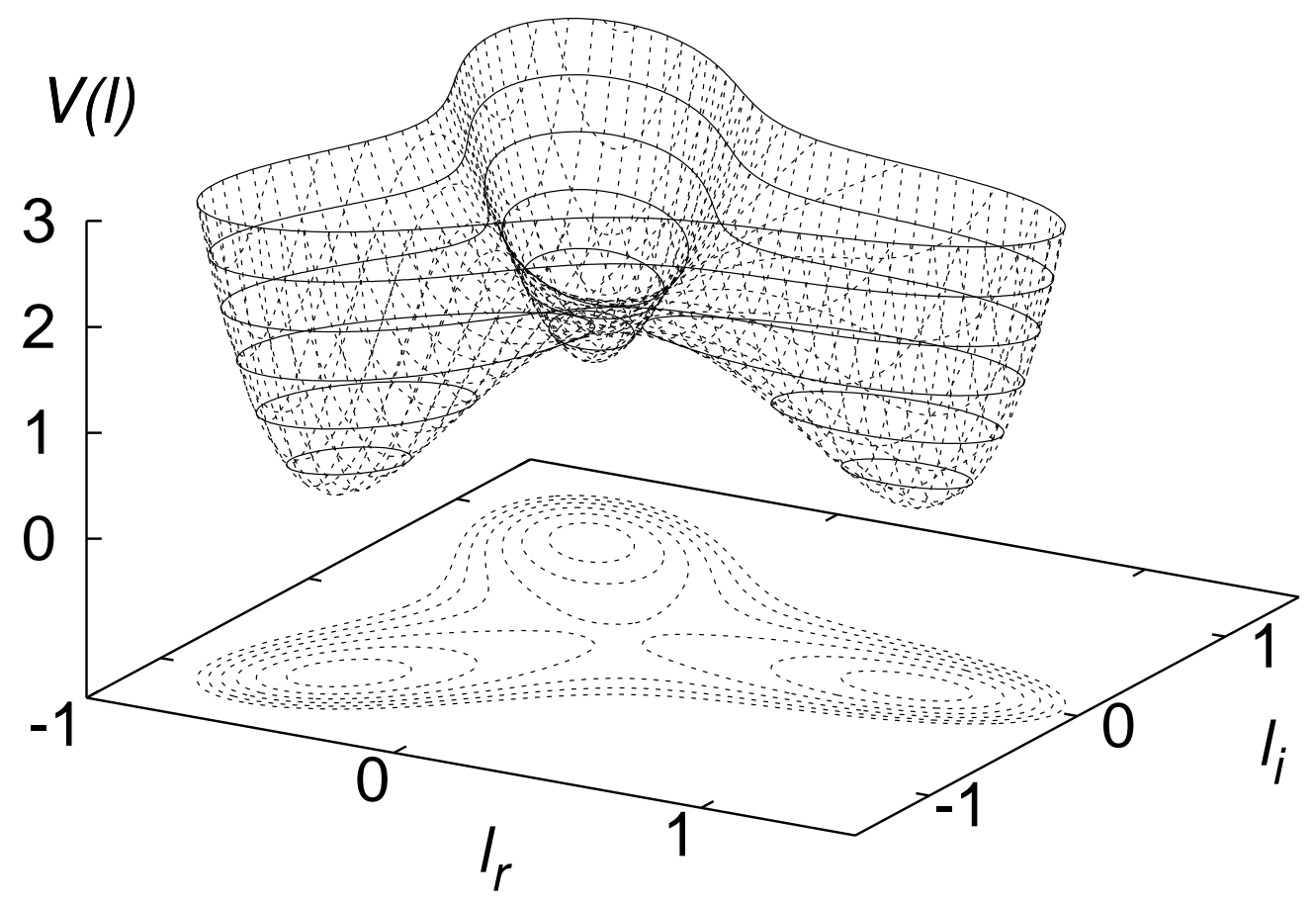

Figure 1: Effective potential for the Polyakov loop $\ell$.

To complete the effective theory in Minkowski space-time, a kinetic term is added:

$$
\mathscr{L}=T^{2}\left(Z_{t}\left|\partial_{t} \ell\right|^{2}-Z_{S}\left|\partial_{i} \ell\right|^{2}\right)-\mathscr{V}(\ell) .
$$

We assume a Lorentz-invariant form, $Z_{t}=Z_{s}$, and take the coefficient $Z_{s}$ from that for spatial variations of SU(3) Wilson lines [10], $Z_{s}=N_{c} / g^{2}$ with $g^{2}=3$. Thus the dynamics of Polyakov loops is in an intermediate regime between very weak $\left(Z_{s} \gg 1\right)$ and very strong $\left(Z_{s} \ll 1\right)$ coupling.

We employ a simulation procedure similar to the one of [12] but focus on heating quenches. Polyakov loop fields are defined on the sites of a spatial cubic lattice of size $N_{s}^{3}$ with periodic boundary conditions. They are initialized in the confined phase at time $t=0$. Then the temperature entering the effective Lagrangian (2.2) is set to a value $T_{f}>T_{c}$ above the deconfinement transition $T_{c}$. The procedure for initializing the field, introducing counterterms in the equations of motion, and coarse graining are described in [7]. The leapfrog algorithm is used to integrate the Euler-Lagrange 
equations, and the system rearranges itself to a new equilibrium ensemble with a non-zero Polyakov loop signaling symmetry breaking in the infinite volume limit.

At time $t$, the structure function is defined by the Fourier transformation of the Polyakov loop:

$$
F(\vec{k}, t)=\frac{a^{3}}{N_{s}^{3}}\left|\sum_{\vec{x}} e^{-i \vec{k} \vec{x}} \ell(\vec{x}, t)\right|^{2} .
$$

For a fixed value of $\vec{k}, F(\vec{k}, t)$ is called SF (structure factors). SFs are our primary observables. In what follows, we label SFs $F_{n}(t)$ as in [8]: $\vec{k}=\vec{n} 2 \pi / L_{s}, n=1: \vec{n}=(1,0,0), \vec{n}^{2}=1, n=2: \vec{n}=$ $(1,1,0), \vec{n}^{2}=2, n=3: \vec{n}=(1,1,1), \vec{n}^{2}=3$, where $L_{s} \equiv a N_{s}$ denotes the size of the lattice in physical units. Note the relation $|\vec{k}|=2 \pi \sqrt{n} / L_{s}$ for $n=1,2,3$. Measurements for $n=1$ include the permutations $(0,1,0),(0,0,1)$ and for $n=2$ the permutations $(1,0,1),(0,1,1)$.

\section{Numerical Results}

In the following we show results from quenches to $T_{f} / T_{c}=1.50$ on $N_{s}=64$ lattices with periodic boundary conditions. Additional results from quenches to larger $T_{f} / T_{c}$ values can be found in Ref. [7]. Our length scale is set by coarse-graining corresponding to the $\mathrm{SU}(3)$ phase transition temperature of $T_{c}=260 \mathrm{MeV}$ and a lattice spacing $a=0.184 \mathrm{fm}$, which gives a physical volume $L_{s}^{3}=\left(a N_{s}\right)^{3}$.

In Fig. \& we present several SF modes from our Glauber dynamics study [8] on a $4 \times 64^{3}$ lattice (quench to $T_{f}=1.57 T_{c}$, average over 170 replica) for comparison with SF modes from the Minkowski dynamics on a $64^{3}$ lattice (quench to $T_{f}=1.50 T_{c}$, average over 200 replica) in Fig. 3. The scale on the vertical axis of Fig. 2 differs from that of Fig. 3 because the former has been determined from the bare Polyakov loop while the effective Lagrangian (2.2) deals with the renormalized loop. Renormalization amounts to a multiplicative constant.

Qualitatively, the SFs display the same behavior: An initial exponential growth is followed by equilibration after the lowest SF mode reaches its maximum. We interpret this as formation of competing order-order domains between regions of different Z(3) triality [6]. For the Minkowskian dynamics the maxima of the lowest SF mode $F_{1}$ are compiled in [7]. They scale with volume in the same way as for Glauber dynamics. Provided the volumes are large enough, fits to the form $F_{1, \max }=b_{0}+b_{1} L_{s}^{3}$ are consistent. Interestingly, both Figs. 2 and 3 show that the above-mentioned effects due to non-perturbatively large variations of the $\mathrm{Z}(3)$ phase in space are visible even for modes with $k \sim T / 2$. This may be related to $Z(3)$ domain walls forming just after the quench being quite broad (disordered phase) and domains not much larger than $1 / T$.

Since the kinetic term in the Lagrangian (2.2) is assumed to be Lorentz invariant, units for time are the same as for length (apart from the speed of light factor $c$ ). When integrating hyperbolic equations, one uses time steps smaller than the spatial lattice spacing $a$. We chose $\Delta t / a=0.01$ and, therefore, in physical units

$$
\Delta t=0.00184 \mathrm{fm} / \mathrm{c} .
$$

We ran trajectories from 15000 to 25000 time steps corresponding to a range from $27.6 \mathrm{fm} / \mathrm{c}$ to $46 \mathrm{fm} / \mathrm{c}$. For the case shown in Fig. 目 the structure factor for the first mode takes about $8 \mathrm{fm} / \mathrm{c}$ to reach its maximum, and another $\approx 20 \mathrm{fm} / \mathrm{c}$ until that mode equilibrates. On the other hand, the 


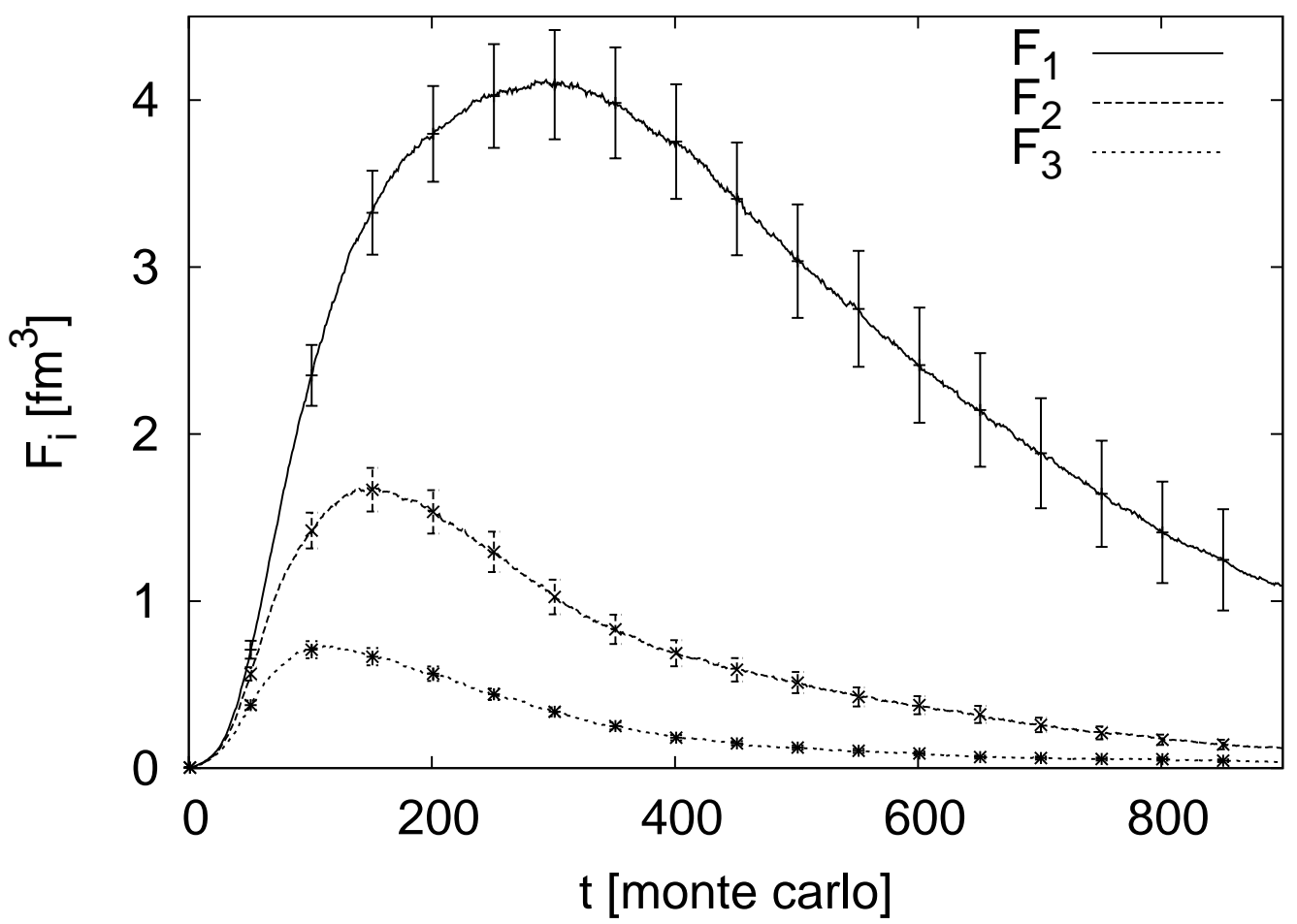

Figure 2: Structure factors for Glauber dynamics [8], $t$ is Monte Carlo time in sweeps $\left(4 \times 64^{3}\right.$ lattice, $T_{f} / T_{c}=1.57$, lattice size $\left.L_{s}=12.1 \mathrm{fm}\right) . F_{n}$ corresponds to $|\vec{k}|=2 \pi \sqrt{n} / L_{s}=\sqrt{n} \times 102.1 \mathrm{MeV}$.

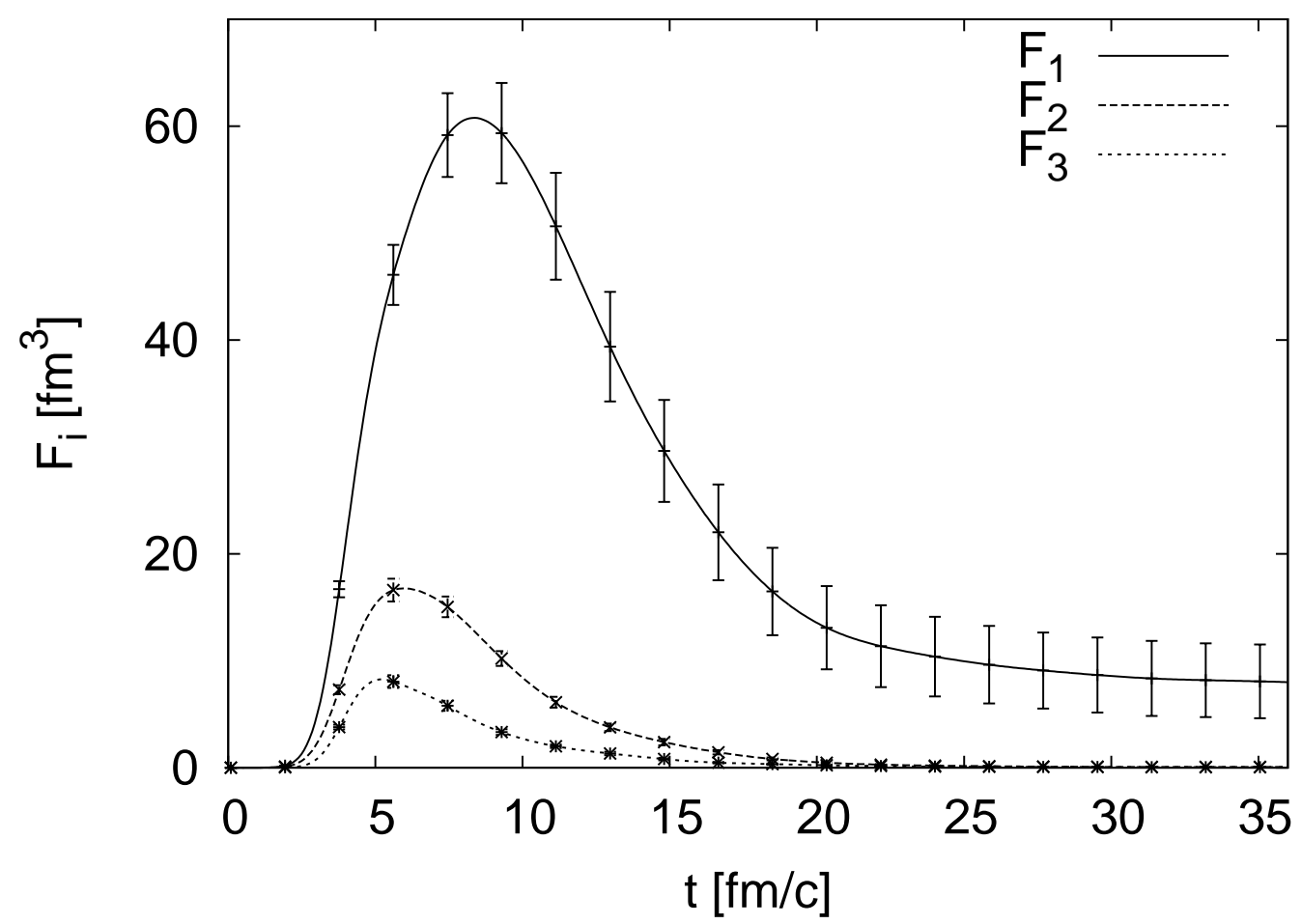

Figure 3: Structure factors for Minkowski dynamics [7], $t$ is real time ( $64^{3}$ lattice, $T_{f} / T_{c}=1.5$, lattice size $\left.L_{s}=11.8 \mathrm{fm}\right) . F_{n}$ corresponds to $|\vec{k}|=2 \pi \sqrt{n} / L_{s}=\sqrt{n} \times 105.3 \mathrm{MeV}$. 
second and third modes grow for a shorter period of time and subsequently equilibrate more rapidly. Note that there is an initial lag, and the precise time for the onset of growth may be sensitive to the spectrum and magnitude of initial fluctuations.

The times $t_{\max }$ needed to reach the maxima of $F_{1}$ for different volumes and quench temperatures are tabulated in Ref. [7]. For the lowest modes of the system they can be quite large, on the order of the size of the system. Also, $t_{\max }$ increases with $T_{f} / T_{c}$ because the barriers between orderorder domains grow higher and are more difficult to overcome (this is different for full QCD where the Z(3) symmetry is broken explicitly). For Glauber dynamics it is known that for sufficiently large systems and corresponding wavelengths $t_{\max }$ scales as $t_{\max }=a_{0}+a_{1} L_{s}^{2}$. For Minkowski dynamics fits to this form are satisfactory too. In the long wavelength limit the equilibration time for a mode with wave vector $k$ would then be proportional to $1 / k^{2}$. However, within our present statistical error bars we cannot exclude a linear dependence, $t_{\max }=a_{0}+a_{1} L_{s}$, either.

\section{Conclusions and Outlook}

The particle spectrum of a quantum field theory at finite temperature builds upon the phase of its vacuum ensemble, and the structure of the vacuum is fundamental for the dynamics of relaxation processes to equilibrium. Heating above $T_{c}$ drives the $\mathrm{SU}(3)$ gauge theory system from the disordered into the ordered phase. The initial process of spinodal decomposition is signaled by an exponential growth of the SFs. Non-perturbatively large variations of the Z(3) phase form domain walls during the conversion from a confined to a deconfined vacuum ensemble. Ordering of the system proceeds through competition of $Z(3)$ domains until one of them eventually occupies the whole system. For realistic temperatures, as relevant for high-energy heavy-ion collisions, we find that the rather slow dynamics of Polyakov loop domains delays thermalization of modes with $k$ nearly up to $T$. In future, it would be interesting to quantify the contribution of these modes to the bulk viscosity.

Our model for Minkowskian dynamics allows one to estimate a physical time scale for the vacuum conversion process. The relaxation times found in this way for $\mathrm{SU}(3)$ pure gauge theory increase as a power of $\sim 1 / k$ for sufficiently low $k$ and are estimated to be on the order of the size $L_{s}$ of the system for $k=2 \pi / L_{s}$ and $L_{s} \approx 10 \mathrm{fm}$. Our numbers should be viewed only as rough first estimates. Nevertheless, it appears that away from the extreme weak-coupling limit $\left(Z_{s} \gg 1\right)$ the dynamics of competing domains will influence thermalization of long wavelength modes, perhaps even for $k$ not very far below $T$, and hence cannot be neglected. The hydrodynamic equations describing the evolution of long-wavelength perturbations in a deconfined medium should therefore be extended to account for the dynamics of competing domains of the Polyakov loop. In the scenario where the system gets stuck before SFs equilibrate, one would like to know as much as possible about phase transitions and equations of state at the relevant non-equilibrium values of the Polyakov loop [13]. E.g., the pressure may then depend on all eigenvalues of the matrix-valued Polyakov loop and not just on their trace.

Acknowledgments: We thank Rob Pisarski for fruitful discussions, and Brookhaven National Laboratory for its hospitality. This work was in part supported by DOE grants DE-FG02-97ER41022, DE-FC02-06ER-41439 and NSF grant 0555397. 


\section{References}

[1] I. Arsene et al. [BRAHMS Collaboration], Nucl. Phys. A 757 (2005) 1; B. B. Back et al., [PHOBOS Collaboration], Nucl. Phys. A 757 (2005) 28; J. Adams et al. [STAR Collaboration], Nucl. Phys. A 757 (2005) 102; K. Adcox et al. [PHENIX Collaboration], Nucl. Phys. A 757 (2005) 184.

[2] See, for example, M. Gyulassy and L. McLerran, Nucl. Phys. A 750 (2005) 30.

[3] R. Baier, A.H. Mueller, D. Schiff and D.T. Son, Phys. Lett. B 502 (2001) 51.

[4] P. Arnold, J. Lenaghan and G.D. Moore, JHEP 0308 (2003) 002.

[5] G. 't Hooft, Nucl. Phys. B 138 (1978) 1; Nucl. Phys. B 153 (1979) 141; A.M. Polyakov, Phys. Lett. B 72 (1978) 477.

[6] T. Bhattacharya, A. Gocksch, C. Korthals Altes and R.D. Pisarski, Nucl. Phys. B 383 (1992) 497; C.P. Korthals Altes, Nucl. Phys. B 420 (1994) 637; C. Korthals-Altes, A. Kovner and M.A. Stephanov, Phys. Lett. B 469 (1999) 205; P. Giovannangeli and C.P. Korthals Altes, Nucl. Phys. B 608 (2001) 203; Nucl. Phys. B 721 (2005) 1; Nucl. Phys. B 721 (2005) 25; P. de Forcrand, M.D. Elia and M. Pepe, Phys. Rev. Lett. 86 (2001) 1438; P. de Forcrand and L. von Smekal, Phys. Rev. D 66 (2002) 011504(R); P. de Forcrand and D. Noth, Phys. Rev. D 72 (2005) 114501; P. de Forcrand, C.P. Korthals-Altes and O. Philipsen, Nucl. Phys. B 742 (2006) 124.

[7] A. Bazavov, B.A. Berg, and A. Dumitru, Phys. Rev. D 78 (2008) 034024.

[8] A. Bazavov, B.A. Berg, and A. Velytsky, Phys. Rev. D 74 (2006) 014501.

[9] T.R. Miller and M.C. Ogilvie, Phys. Lett. B 488 (2000) 313; Nucl. Phys. B (Proc. Suppl.) 94 (2001) 419.

[10] R.D. Pisarski, Phys. Rev. D 62 (2000) 111501(R); A. Dumitru and R.D. Pisarski, Phys. Lett. B 504, 282 (2001); Phys. Rev. D 66 (2002) 096003.

[11] A.J. Mizher, E.S. Fraga, G. Krein, Braz. J. Phys. 37 (2007) 605; E.S. Fraga, G. Krein and A.J. Mizher, Phys. Rev. D 76 (2007) 034501; N.C. Cassol-Seewald, R.L.S. Farias, E.S. Fraga, G. Krein, and R.O. Ramos, arXiv:0711.1866v1.

[12] O. Scavenius, A. Dumitru, and A.D. Jackson, Phys. Rev. Lett. 87 (2001) 182302.

[13] E. Shuryak, arXiv:0807.3033, section 3.4. 